\title{
EVALUACIÓN DE LA VACUNA COMPLEJO VIRUS - ANTICUERPO ADMINISTRADA in ovo VERSUS UN PROGRAMA TRADICIONAL CONTRA LA ENFERMEDAD INFECCIOSA DE LA BURSA BAJO CONDICIONES DE CAMPO EN POLLOS DE ENGORDE
}

\author{
Evaluation of the Virus-Antibody Complex Vaccine Administered in ovo \\ Versus a Traditional P rogramme Against the Infectious Bursal Disease \\ Under Field CONditions in B RoILERS
}

\section{Carmen Paredes V. ${ }^{1}$, Mónica Alba Ch. ${ }^{1}$, Alberto Manchego S. ${ }^{2}$, Rosa Perales C. $^{3}$ y Pablo Reyna S. ${ }^{1,4}$}

\section{Resumen}

\begin{abstract}
Se evaluó un nuevo programa de vacunación contra la Enfermedad Infecciosa de la Bursa que contenía la vacuna complejo virus-anticuerpo en comparación con un programa tradicional bajo condiciones de campo. En cada grupo se utilizó 21,800 pollos de engorde de ambos sexos de la línea Cobb Vantress 500. El grupo experimental recibió un complejo virus-anticuerpo en la planta de incubación a los 18 días de desarrollo embrionario, y el grupo control recibió dos vacunas a virus vivo, Bursine ${ }^{\circledR} 2$ y Bursa Blen, administradas por agua de bebida a los 8 y 19 días de edad, respectivamente. Se determinó en forma semanal y hasta los 47 días de edad, en ambos grupos, el peso corporal, índice de conversión alimenticia (ICA), índice de eficiencia productiva europeo (IEPE), mortalidad, índice bursal, lesiones histopatológicas y niveles de anticuerpos contra el VEIB. Las aves del grupo experimental tuvieron un mejor peso corporal a los 47 días de edad ( $\mathrm{p}<0.05$ ), ICA y IEPE. Ambos grupos mostraron atrofia bursal a partir de los 35 días de edad; sin embargo, los resultados microscópicos mostraron lesiones moderadas de atrofia y depleción linfoide en las bursas del grupo experimental a los 35 días de edad. Los títulos de anticuerpos fueron mayores a los 35 y 42 días de edad en el grupo experimental y en el grupo control a los 47 días $(\mathrm{p}<0.05)$. Ambos programas produjeron lesiones de grado variable en las bursas con la estimulación de una respuesta inmune activa; sin embargo, las aves que recibieron la vacuna virus-anticuerpo tuvieron un mejor desempeño productivo.
\end{abstract}

Palabras clave: Enfermedad Infecciosa de la Bursa, índice bursal, vacuna virus-anticuerpo, programa de vacunación tradicional, vacunación in ovo, parámetros productivos

\footnotetext{
${ }^{1}$ Laboratorio de Patología Aviar, ${ }^{2}$ Laboratorio de Microbiología y Parasitología Veterinaria, ${ }^{3}$ Laboratorio de Histopatología, Embriología y Patología Veterinaria, Facultad de Medicina Veterinaria, Universidad Nacional Mayor de San Marcos, Lima

${ }^{4}$ E-mail: reynapa2002@yahoo.com
} 
A new vaccination programme against infectious bursal disease using a virusantibody complex vaccine in comparison with a traditional programme under field conditions was evaluated. In each group, 21,800 broiler chickens of Cobb Vantress 500 line were used. The experimental group was vaccinated with the strain 2512 in combination with specific antibodies at the incubation plant on day 18 of embryonic development, whereas the control group received two vaccines of live virus, the Bursine ${ }^{\circledR} 2$ and Bursa Blen, administered by drinking water at 8 and 19 days of age respectively. Body weight, feed conversion index, European Production Efficiency Index, mortality, bursal index, histopathological lesions, and antibody levels against IBDV were evaluated. Birds of the experimental group showed higher body weight $(\mathrm{p}<0.05)$, and better feed conversion index and production efficiency index at 47 days of age than the control group. Both groups showed bursal atrophy from 35 days of age onwards, however, the histopathological results only showed moderate lesions of atrophy and lymphoid depletion in bursas in both groups during those periods. Antibody titers were higher at 35 and 42 days of age in the experimental group and at 47 days in the control group $(p<0.05)$. Both vaccination programmes produced lesions of variable degree in bursas, nevertheless, birds that received the vaccine complex virus antibody had a better productive performance.

Key words: Infectious Bursal Disease, bursal index, virus-antibody vaccine, traditional vaccination programme, in ovo vaccination, productive parameters

\section{INTRODUCCIÓN}

La Enfermedad Infecciosa de la Bursa (EIB) es una enfermedad viral aguda, altamente contagiosa que afecta a las aves jóvenes (Rosales, 1997) y cuyo agente etiológico pertenece a la familia Birnaviridae, género Avibirnabirus (Lasher y Shane, 1994; Hafez, 2002; Luckert y Saif, 2003). Es un virión sin envoltura con cubierta sencilla de 55-65 nm de diámetro y de simetría icosahédrica (Morales, 1993; Lasher y Shane, 1994; van den Berg y Eterradossi, 2001). La enfermedad se caracteriza por la destrucción de linfocitos en la bursa de Fabricio y, en menor grado, en otros tejidos linfoides (Butcher y Miles, 1993; Hafez, 2002; Luckert y Saif, 2003). La manifestación de la enfermedad ha dado lugar a la emergencia de cepas variantes y cepas altamente virulentas que han cambiado drásticamente la epidemiología de esta enfermedad (van den Berg, 2000).
La importancia económica de esta enfermedad se manifiesta tanto de forma directa sobre la productividad, como por las consecuencias de la inmunosupresión que produce en las aves (Morales, 1995). La forma clínica de la enfermedad se observa en aves de tres semanas a más, caracterizada por depresión y diarreas; reducción en la ganancia de peso e incremento en la conversión alimenticia, tasa de mortalidad y decomisos (Hafez, 2002). La segunda forma de la EIB es la más importante, y se manifiesta por una inmunosupresión grave y prolongada en los pollos infectados a una edad temprana (Luckert y Saif, 2003), dando como resultado una mayor susceptibilidad a una gran variedad de agentes infecciosos (van der Sluis, 1995).

La avicultura tecnificada ha respondido a la EIB con la adopción de medidas de bioseguridad y el uso intensivo de vacunas vivas, clasificadas según su patogenecidad en suaves, intermedias y fuertes; sin embargo, 
la EIB continua causando importantes pérdidas económicas (Rosales, 1993; Yehoshua, 1999; Villegas, 2002). El principal problema en la inmunización contra la EIB es la desuniformidad en el nivel de anticuerpos maternales en la progenie ya que la vacunación temprana puede ser neutralizada por los altos niveles de anticuerpos maternales; por esta razón, se requiere repetir la vacunación durante las primeras semanas de vida (Morales, 1993; Tsukamoto et al., 1995; Villegas, 2002; Rautenschlein et al., 2003).

En el Perú, el programa de vacunación tradicional en pollos de carne contra la EIB es de dos vacunas vivas de patogenicidad intermedia administradas en campo. Esta práctica ha dado buenos resultados; sin embargo, se han presentado problemas de inmunosupresión, probablemente relacionados a la EIB; de allí la necesidad de desarrollar nuevas alternativas de vacunación y de vacunas eficaces para la producción avícola comercial (Hassan et al., 1996). Dentro de estas, la vacunación in ovo es un método relativamente nuevo para la administración de vacunas, que actualmente se viene realizando contra la enfermedad de Marek (Villegas, 1991). Este es un método basado en el desarrollo de madurez inmunológica antes del nacimiento de la aves (Hafez, 2002).

La búsqueda de nuevas tecnologías ha dado lugar al surgimiento de un nuevo tipo de vacuna contra la EIB a base de virus vivo mezclado con antisuero específico, para formar una vacuna a base de un complejo virusanticuerpo (Whitfill et al., 1995, 2002; van den Berg, 2000; Avakian et al., 2001; van den Wijngaard et al., 2001). Al parecer, la presencia de los anticuerpos maternales en contra del VEIB no interfieren con la habilidad de esta vacuna de estimular la producción de una respuesta inmune capaz de proteger al ave (Whitfill et al., 2002).

La aplicación de la vacuna virus-anticuerpo contra la EIB constituye una alternativa en el país y, por lo tanto, es materia de inves- tigación. El presente estudio tuvo como objetivo medir la eficacia de esta vacuna administrada in ovo frente a un programa de vacunación tradicional contra la EIB bajo condiciones de campo en pollos de engorde.

\section{Materiales y Métodos}

\section{Lugar de Estudio}

La crianza de las aves se realizó en las instalaciones de una empresa avícola privada, localizada en el distrito de Guadalupe, departamento de Ica. La prueba serológica y el estudio histopatológico se realizaron en el Laboratorio de Patología Aviar y el Laboratorio de Histopatología, Embriología y Patología Veterinaria de la Facultad de Medicina Veterinaria de la Universidad Nacional Mayor de San Marcos, respectivamente.

\section{Aves}

Se utilizaron 43,600 pollos de carne de un día de edad de la línea Cobb Vantress 500, provenientes de los lotes de reproductores de la misma empresa comercial. Las aves se distribuyeron en un grupo control que recibió un programa de vacunación tradicional contra el VEIB, administrado en el campo; y un grupo experimental, que recibió una sola vacuna contra el VEIB, administrada en la planta de incubación. En cada grupo se distribuyó 21,800 pollos $(10,800$ machos y 11,000 hembras).

\section{Vacunas}

Se utilizaron vacunas comerciales a virus vivo: Bursaplex $\mathrm{IBD}^{\circledR}$ (Embrex) conteniendo el Complejo Ag/Ac con la cepa 2512 (Intermedia plus); Bursine ${ }^{\circledR} 2$ (Fort Dodge, Animal Health) con la cepa Lukert (Intermedia suave); y Bursa Blen (Ceva) con la cepa 2512 (Intermedia intermedia). Adicionalmente, todas las aves fueron vacunadas contra la Enfermedad de Newcastle y Bronquitis Infecciosa, en la planta de 
incubación, y a los 8 y 19 días de edad, con las vacunas Nobilis B1 La Sota y Cevac ${ }^{\circledR}$ VitaBron L vía agua de bebida.

\section{Diseño Experimental}

? Grupo Experimental: aves vacunadas con el Complejo Ag/Ac Bursaplex ${ }^{\circledR}$ a los 18 días de incubación vía saco alantoideo, mediante un sistema automatizado en planta de incubación.

? Grupo Control: aves vacunadas con un programa tradicional a los 8 y 19 días de edad con Bursine ${ }^{\circledR} 2$ y Bursa Blen, respectivamente, vía agua de bebida.

\section{Parámetros Productivos}

Peso vivo. Se registró semanalmente y conjuntamente con el consumo del alimento, hasta el final del experimento, para calcular el índice de conversión.

Índice de Conversión Alimenticia (ICA). Se obtuvo en base a la siguiente fórmula:

$$
\mathrm{ICA}=\frac{\text { Consumo de alimento }(\mathrm{kg})}{\text { Peso corporal }(\mathrm{kg})}
$$

Mortalidad. Se registró diariamente, realizándose la necropsia a todas las aves muertas.

Índice de Eficiencia Productivo Europeo $(I E P E)$. ). Se usó para evaluar el rendimiento integral de cada grupo mediante la fórmula:

\section{IEPE $=\underline{\text { Viabilidad }(\%) \times \text { Ganancia de peso/día }} \times 100$ ICA}

Evaluación del Índice Bursal. Para la evaluación macroscópica de la bursa de Fabricio se utilizó el Índice Bursal (IB), para lo cual, se sacrificaron 10 aves por grupo a los 7, 14, $21,28,35,42$ y 47 días de edad. El IB se obtuvo siguiendo la recomendación de Giambrone (1987):

$$
\mathrm{IB}=\frac{\text { Peso de la bursa }(\mathrm{g})}{\text { Peso corporal }(\mathrm{g})} \times 1000
$$

donde:

$1.5-3.5=$ Bursa normal

$0.5-1.5=$ Atrofia bursal

$<0.5=$ Severa atrofia bursal

Evaluación microscópica de las lesiones de la bursa. Se tomaron muestras de bursa de 5 aves por grupo a los 7, 14, 28, 35 y 42 días de edad para su examen histopatológico. Los resultados se expresaron como el promedio de las lesiones halladas por grupo. La evaluación histopatológica de la bursa se realizó de acuerdo al método de Skeeles (2001) y Rosales et al. (1989), quienes emplearon un sistema de calificación cualitativo y cuantitativo, respectivamente. Ambos métodos se basan en una escala de grados de lesiones del 1 al 4:

(1) No se observan lesiones, estructura folicular normal (Bursa normal).

(2) Leve depleción linfoide en folículos linfoides (5 - 25\% de folículos afectados).

(3) Moderada atrofia o depleción linfoide (26 - 74\% de folículos afectados).

(4) Severa atrofia difusa folicular (75-100\% de folículos afectados).

Evaluación serológica. $\mathrm{Al}$ azar, 50 aves de cada grupo experimental fueron sangradas mediante punción alar a los 1, 7, 14, 21, 28, 35,42 y 47 días de edad, para determinar los niveles de anticuerpos contra el VEIB. Esta medición se realizó utilizando la prueba serológica comercial de ELISA indirecta usando un kit estándar (IDEXX Laboratories, Inc.Westbrook, ME). Los resultados serológicos se expresaron como promedios geométricos (PGT) y coeficiente de variación (CV) de acuerdo a métodos descritos por Wyeth (1975).

\section{Análisis Estadístico}

Las pruebas de Análisis de Varianza (ANOVA) y "t" Student se utilizaron para determinar diferencias estadísticas en los resultados de peso corporal y respuesta serológica entre grupos, respectivamente. La 
Cuadro 1. Comportamiento productivo de los pollos de carne de la línea Cobb Vantress 500 sometidos a dos programas de vacunación contra la Enfermedad Infecciosa de la Bursa

\begin{tabular}{lccccc}
\hline Grupos $^{1}$ & Sexo & $\begin{array}{c}\text { Peso corporal } \\
(\mathrm{kg} ? \text { ee })\end{array}$ & $\mathrm{ICA}^{2}$ & $\begin{array}{c}\text { Mortalidad } \\
(\%)\end{array}$ & IEPE $^{3}$ \\
\hline \multirow{2}{*}{ Control } & Macho & $2.692^{\mathrm{a}} ? 0.033$ & 1.89 & $8.79 \mathrm{a}$ & 276.41 \\
& Hembra & $2.283^{\mathrm{b}} ? 0.018$ & 2.03 & $6.02 \mathrm{~b}$ & 224.88 \\
& Macho & $2.807^{\mathrm{c}} ? 0.028$ & 1.85 & $6.15 \mathrm{c}$ & 302.98 \\
Experimental & Hembra & $2.352^{\mathrm{d}} ? 0.037$ & 1.95 & $6.46 \mathrm{~b}$ & 240.05 \\
\hline
\end{tabular}

a,b,c,d $(p<0.05)$

${ }^{1}$ Grupo control: Bursine? 2 a los 8 días y Bursa Blen a los 19 días de edad, ambas en el agua de bebida; Grupo experimental: Vacuna complejo virus-anticuerpo mediante vacunación in ovo el día 18 de incubación.

${ }^{2}$ Índice de Conversión Alimenticia

3 Índice de Eficiencia Productiva Europeo

Cuadro 2. Índice bursal ${ }^{1}$ por días de edad en pollos de carne de la línea Cobb Vantress 500 sometidos a dos programas de vacunación contra la Enfermedad Infecciosa de la Bursa

\begin{tabular}{lccccccc}
\hline & \multicolumn{7}{c}{ Edad (días) } \\
\cline { 2 - 8 } Grupos $^{2}$ & 7 & 14 & 21 & 28 & 35 & 42 & 47 \\
\hline Control & 1.10 & 1.93 & 2.48 & 1.64 & 0.98 & 0.56 & 0.45 \\
Experimental & 1.25 & 2.12 & 2.04 & 2.08 & 0.68 & 0.53 & 0.46 \\
\hline
\end{tabular}

${ }^{1}$ Según Giambrone (1987)

${ }^{2}$ Grupo control: Bursine? 2 a los 8 días y Bursa Blen a los 19 días de edad, ambas en el agua de bebida; Grupo experimental: Vacuna complejo virus-anticuerpo mediante vacunación in ovo el día 18 de incubación

prueba de Chi Cuadrado se utilizó para determinar la asociación estadística entre los resultados de mortalidad de los dos grupos.

\section{Resultados}

En el Cuadro 1 se muestra los resultados del peso corporal, ICA, mortalidad e
IEPE de los dos grupos en estudio, distribuidos por sexo. El peso corporal en las aves del grupo experimental fue significativamente mayor al del grupo control $(\mathrm{p}<0.05)$. La mayor tasa de mortalidad ocurrió en los machos del grupo control, seguido de las hembras del grupo experimental. El mejor resultado del ICA y del IEPE se observó en las aves del grupo experimental. 
Cuadro 3. Calificación semanal de las lesiones microscópicas de la bursa ${ }^{1}$ de pollos de carne de la línea Cobb Vantress 500 sometidos a dos programas de vacunación contra la Enfermedad Infecciosa de la Bursa

\begin{tabular}{lccccc}
\hline & \multicolumn{5}{c}{ Edad (días) } \\
\cline { 2 - 6 } Grupos $^{2}$ & 7 & 14 & 28 & 35 & 42 \\
\hline Control & 1.75 & 2.00 & 3.25 & 3.67 & 3.00 \\
Experimental & 2.00 & 2.00 & 2.00 & 3.33 & 3.60 \\
\hline
\end{tabular}

${ }^{1}$ Adaptado según Skeeles (2001) y Rosales et al. (1989)

${ }^{2}$ Grupo control: Bursine? 2 a los 8 días y Bursa Blen a los 19 días de edad, ambas en el agua de bebida; Grupo experimental: Vacuna complejo virus-anticuerpo mediante vacunación in ovo el día 18 de incubación.

Cuadro 4. Promedio geométrico de títulos de anticuerpos (PGT) y coeficiente de variación (CV) semanal contra el VEIB por la prueba de ELISA en pollos de carne de la línea Cobb Vantress 500 sometidos a dos programas de vacunación

\begin{tabular}{rcccccccc}
\hline \multirow{2}{*}{ Grupos $^{1}$} & \multicolumn{8}{c}{ Edad (días) } \\
\cline { 2 - 9 } & 1 & 7 & 14 & 21 & 28 & 35 & 42 & 47 \\
\hline Control & $4844^{\mathrm{a}}$ & $3012^{\mathrm{a}}$ & $715^{\mathrm{a}}$ & $154^{\mathrm{b}}$ & $208^{\mathrm{a}}$ & $1492^{\mathrm{a}}$ & $1832^{\mathrm{b}}$ & $2574^{\mathrm{a}}$ \\
$(\mathrm{CV})^{2}$ & $(31.8)$ & $(19.4)$ & $(57.2)$ & $(58.0)$ & $(114.4)$ & $(57.0)$ & $(34.5)$ & $(39.5)$ \\
Experimental & $4985^{\mathrm{a}}$ & $3011^{\mathrm{a}}$ & $764^{\mathrm{a}}$ & $258^{\mathrm{a}}$ & $144^{\mathrm{b}}$ & $2045^{\mathrm{b}}$ & $2305^{\mathrm{a}}$ & $2305^{\mathrm{b}}$ \\
$(\mathrm{CV})$ & $(26.0)$ & $(30.6)$ & $(52.7)$ & $(49.0)$ & $(44.9)$ & $(34.4)$ & $(27.6)$ & $(27.4)$ \\
\hline
\end{tabular}

$a, b(p<0.05)$

'Grupo control: Bursine' 2 a los 8 días y Bursa Blen a los 19 días de edad, ambas en el agua de bebida; Grupo experimental: Vacuna complejo virus-anticuerpo mediante vacunación in ovo el día 18 de incubación.

${ }_{2}^{2}$ (CV): Coeficiente de variación

El índice de peso bursal se presenta en el Cuadro 2. Se observó un incremento gradual del IB durante las primeras tres semanas de edad, el cual podría estar relacionado con el incremento de peso de la bursa. Ambos grupos presentaron un IB normal hasta los 28 días de edad, pero con una tendencia de atrofia bursal en el grupo control, manifestándose en forma evidente en ambos grupos a los 35 días de edad, y llegando a la atrofia severa en los dos grupos a los 47 días de edad.
Los resultados de calificación de lesiones microscópicas de la bursa se muestran en el Cuadro 3. Las lesiones bursales de los pollos del grupo control se incrementaron hasta los 28 días, mientras que en el grupo experimental se incrementaron hasta los 35 días de edad.

Los niveles de anticuerpos maternales contra el VEIB fueron muy similares al primer día de edad en ambos grupos. Luego se 
observó una disminución durante las primeras 3-4 semanas de edad en ambos grupos. Los títulos de anticuerpos contra el VEIB por efecto de los programas de vacunación comenzaron a incrementarse a partir de los 35 días de edad, siendo estadísticamente mayores los del grupo experimental $(\mathrm{p}<0.05)$.

\section{Discusión}

En el presente estudio se reveló que las aves con vacuna antígeno-anticuerpo ganaron $3.7 \%$ más de peso, la ventaja en la conversión alimenticia fue de $3.15 \%$, y adicionalmente la supervivencia fue $1.1 \%$ más elevada. Asimismo, al combinar la ganancia de peso, porcentaje de supervivencia, edad final del experimento y conversión alimenticia dentro de un solo índice productivo, la ventaja observada fue $20.87 \%$ para el grupo control. Estos parámetros concuerdan con otros reportes para ganancia de peso (Whitfill et al., 2002), ICA y porcentaje de mortalidad (Whitfill et al., 2002; Li et al., 2005), e IEPE (Szelesczuk et al., 2001; Li et al.,2005).

Ambos grupos mostraron un índice bursal normal hasta los 28 días de edad, pero a los 35 días disminuyó drásticamente, mostrando una atrofia bursal de acuerdo a la clasificación de Giambrone (1987), y similar a los resultados reportados por Haddad et al. (1997) y Avakian et al. (2001), que han utilizado la vacuna virus-anticuerpo.

El estudio histopatológico reveló lesiones leves similares en ambos grupos a los $7 \mathrm{y}$ 14 días de edad. Una vez que el virus de la vacuna sobrepasa la inmunidad maternal comienza la replicación viral (Avakian et al., 2001; Whitfill et al., 2002). El virus vacunal de la vacuna virus-anticuerpo alcanza la bursa más rápido en aquellas aves que poseen bajos títulos de anticuerpos maternales (Jeurissen et al., 1998; Avakian et al., 2001); sin embargo, se indica que no causa lesiones microscópicas a la bursa hasta los 6 - 8 días después de la vacunación de pollos SPF (Whitfill et al., 1995, 2002).
Los dos grupos mostraron lesiones microscópicas de atrofia bursal desde moderada a severa, a los 35 días de edad; sin embargo, estas lesiones ya se observaban desde el día 28 en el grupo control. Otros reportes sobre el uso de la vacuna virus-anticuerpo han mostrado cambios histológicos en las bursas a los 35 días de edad, como atrofia folicular difusa, depleción linfoide cortical y medular, infiltración de macrófagos, y repliegues del epitelio (Haddad et al., 1997); lesiones que se asemejan a las halladas en este estudio. La presentación de severa atrofia bursal en la evaluación microscópica ha sido reportado con el uso de la cepa intermedia 2512, vía agua de bebida (Mazariegos et al., 1990; Gallardo, 1998).

Los títulos de anticuerpos al primer día de edad evidenciaron protección y uniformidad de las aves en ambos grupos. Los anticuerpos maternales para el VEIB descendieron durante las primeras 3-4 semanas de edad, seguida del inicio de la respuesta inmune activa humoral que comenzó hacia el día 35 en ambos grupos; sin embargo, las aves del grupo experimental mostraron una mayor respuesta inmune humoral que las aves del grupo control, obteniendo títulos significativamente más altos a los 35 y 42 días. Estos resultados no coinciden totalmente con otros autores quienes señalan títulos de anticuerpos más altos contra el VEIB al final de campaña en las aves que recibieron la vacuna virus-anticuerpo (Johnston et al., 1997; Baines y van den Wijngaard, 1999; Avakian et al., 2001). Los estudios realizados por Corley et al. (2002) y Avakian et al. (2001) demostraron que aves con anticuerpos maternales vacunadas con la vacuna virus-anticuerpo in ovo no tuvieron una respuesta de anticuerpos a los 21 días post vacunación, pero las aves resultaron protegidas de atrofia bursal cuando fueron desafiadas en ese momento. Esta resistencia al desafío en ausencia de anticuerpos implica que la inmunidad celular estaría involucrada en la protección contra el VEIB. 
Según los resultados obtenidos en el presente experimento, las aves que recibieron la vacuna virus-anticuerpo tuvieron un mejor desempeño productivo, una mejor protección de la bursa y un menor índice bursal; no obstante, ambos programas lograron producir una respuesta inmune activa no evidenciándose la forma clínica de la enfermedad en este estudio.

\section{Conclusiones}

? Las aves que recibieron la vacuna virus-anticuerpo obtuvieron los mejores resultados productivos.

? Los grupos que recibieron la Cepa tipo Lukert a los 8 días y la cepa Winterfield 2512 a los 19 días de edad, vía agua de bebida, y el grupo que recibió la vacuna complejo virus-anticuerpo mediante vacunación in ovo el día 18 de incubación mostraron atrofia bursal de tipo leve como consecuencia de la vacunación.

\section{Literatura Citada}

1. Avakian AP, Craig WE, Haddad EE. 2001. The characteristic of infectious bursal disease virus - Antibody complex caccines and their application in broilers with maternal immunity. Proc Cost Action 839 «Inmunosupresive Viral Disease in Poultry», Pulawi, Poland.

2. Baines $D$, van den Wijngaard JK. 1999. State of the art in ovo technology. Int Hatchery Practice 13(6): 15-17.

3. Butcher G, Miles R. 1993. Prevención y control de gumboro. Industria Avícola 40(7): 8-10.

4. Corley MM, Giambrone JJ, Dormitorio $T V .2002$. Evaluation of the immune response and detection of Infectious Bursal Disease viruses by reverse transcriptase - polymerase chain reaction and enzyme-linked immunosorbent assay after in ovo vaccination of commercial broilers. Avian Dis 46: 803-809.
5. Gallardo W. 1998. Evaluación serológica e histopatológica de pollos broilers vacunados contra la enfermedad de Gumboro a los 12 días de edad. Tesis de Médico Veterinario. Lima: Facultad de Medicina Veterinaria, Univ. Nacional Mayor de San Marcos. 38 p.

6. Giambrone J. 1987. Evaluación y relaciones morfométricas en la enfermedad infecciosa de la bursa como método de diagnóstico. Georgia, USA: The American of Avian Pathology. p 24-38.

7. Haddad EE, Whitfill CE, Avakian AP. 1997. Efficacy of a novel infectious bursal disease virus immune complex vaccine in broilers chickens. Avian Dis 41: 882-889.

8. Hafez M. 2002. Infección de la bolsa de Fabricio. Evolución de la enfermedad de Gumboro e innovaciones para su control. X Curso de Actualización de Avimex. México. p 7-18.

9. Hassan MK, Al-Natour MQ, Saif YM. 1996. Pathogenecity, attenuation, and immunogenecity of Infectious Bursal Disease virus. Avian Dis 40: 567-571.

10. Jeurissen SH, Janse EM, Lehrbach PR, Haddad EE, Avakian A, Whitfill CE. 1998. The working mechanism of an immune complex vaccine against infectious bursal disease. Immunology 95: 494-500.

11. Johnston PA, Liu H, O'Connell T, Bland M, Tyczkowsky J, Kemper A. 1997. Applications in ovo technology. Poultry Sci 76: 165-178.

12. Lasher HN, Shane SM. 1994. Infectious Bursal Disease. World Poultry Sci 50: 133-158.

13. Li CS, Wang LY, Chou CC. 2005. Field evaluation of flock production performance of in ovo injection of Infectious Bursal Disease virus immune complex vaccine in commercial broilers farms. J Appl Poult Res 14: 338-344.

14. Luckert PD, Saif VM. 2003. Infectious bursal diseases. In: Calnek B, Barnes H, (eds). Disease of poultry $11^{\text {th }}$ ed. Iowa: Iowa State University Press. p 161-174. 
15. Mazariegos LA, Luckert PD, Brown J. 1990. Pathogenecity and immunosuppressive properties of Infectious Bursal Disease «intermediate» strains. Avian Dis 34: 203-208.

16. Morales O. 1993. La enfermedad de Gumboro. Mundo Avícola 8: 21-24.

17. Morales O. 1995. Actualidades en el control de la enfermedad infecciosa de la bolsa. Seminario Técnico Avícola. Memorias AMEVEA. Santa Cruz, Bolivia. $\mathrm{p}$ 69-70.

18. Rautenschlein S, Yeh HY, Sharma JM. 2003. Comparative immunopathogenesis of mild, intermediate, and virulent strains of classic infectious bursal disease virus. Avian Dis 47: 66-78.

19. Rosales G. 1997. Control de la infección de la bolsa de Fabricio. Acontecer Avícola 5(22): 27-31.

20. Rosales AG, Villegas $P$, Luckert PD, Fletcher OJ, Mohamed MA, Brown J. 1989. Isolation, identification, and pathogenicity of two field strains of infectious bursal disease virus. Avian Dis 33: 35-41.

21. Rosales G. 1993. Aspectos de campo en el control de la Enfermedad Infecciosa de la Bolsa de Fabricio en reproductoras y pollos de engorde. III Congreso Nacional de avicultura (APA). Trujillo, Perú. p 67-69.

22. Skeeles JK. 2001. Score de lesiones bursales. Boletín SHERING-PLOUGH Animal Health. Multivet.

23. Tsukamoto K, Tanimura N, Kakita S, Ota K, Mase M, Imai K, Hihara T. 1995. Efficacy of three live vaccines against highly virulent infectious bursal disease virus in chickens with or without maternal antibodies. Avian Dis 39: 218-229.
24. van der Sluis W. 1995. Infectious bursal disease virus: destructor of the immune system. World Poultry (Suppl.): 4-6.

25. van den Berg T. 2000. Acute infectious bursal disease in poultry: a review. Avian Pathol 29: 175-194.

26. van den Berg T, Eterradossi N. 2001. Doenca infecciosa bursal aguda em aves. II Simposio da Doenca de Gumboro. Campinas, Brasil. p 103-122.

27. van den Wijngaard JK, Avakian A, Craig W, Eid H. 2001. La vacunación in ovo contra la EIB sobrepasa el enfoque convencional. World Poultry 14: 13-15.

28. Villegas P. 1991. Vacunación in ovo: Perspectivas. Avicultura Profesional 8(4): 153.

29. Villegas P. 2002. Enfermedad infecciosa de la bolsa. Industria Avícola 49(7): 22-25.

30. Whitfill CE, Haddad EE, Ricks CA, Skeeles JK, Newberry LA, Beasley $J N$, Andrews PD, Thoma JA, Wakenell PS. 1995. Determination of optimum formulation of a novel infectious bursal disease virus (IBDV) vaccine constructed by mixing bursal disease antibody with IBDV. Avian Dis 39: 687-699.

31. Whitfill CE, Avakian AP, Haddad EE, Wakenell PS. 2002. Vacunas con el complejo virus anticuerpo: presente y futuro. Avicultura Profesional 20(1): 17-22.

32. Yehoshua S. 1999. Importancia de agentes inmunosupresores en avicultura. Mundo Avícola y Porcino 32: 1-22.

33. Wyeth PJ. 1975. Effect of infectious bursal disease on the response of chickens to S. typhymurium and E. coli infection. Vet Rec 96: 238-243. 